\title{
Regulated bioanalytical laboratories: technical and regulatory aspects from global perspectives
}

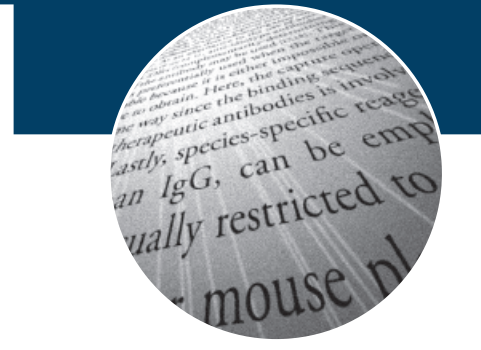

\section{"Regulatory science is still like watching paint dry, but we must get the best data that we can and, indeed, paint must dry."}

Most of us who pursue academic degrees in analytical chemistry have only a vague notion of the regulatory formalities that await us in the real world. Once we find out about them, a few will say and many more will think, 'I did not get a research degree to be encumbered by all this. It is worse than watching paint dry or filing tax returns.' There are three reasons we are subject to these regulations. First, those who came before us were occasionally sloppy in the laboratory and in writing reports. Second, our predecessors have also been caught making things up and thus could not be trusted and finally, the data we are responsible for obtaining is very important to making decisions that impact the lives of humans, animals and shareholders. There have been too many examples in the history of science where scientists demonstrated that they could not be fully trusted to provide accurate data. While this has long been true of finance people, historians and politicians, science was once thought to be reliably based on integrity. In response to these challenges, demands were made on government monitors, and the GXP rules of the road evolved to the present day. I will admit that I find this all very unpleasant as do most of my academic colleagues and discovery people at the tip of the spear of new pharmaceutical science. On the other hand, I find it to be very important. Given a chance; bias, neglect and willful malfeasance are an inevitable side-effect whenever humans make measurements.

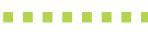 \\ "I have been using this volume for 3 months and have referred to it frequently. Zhou has done a good job that few others would attempt."}

The current volume approaches 600 pages and primarily deals with obtaining bioanalytical data in the regulated environment associated with toxicology and clinical trials. The vastness of regulatory science is evidenced by the fact that the bioanalytical component is a small one when matched with corresponding preclinical, clinical and manufacturing components. The principles of independent quality assurance of the systems to control quality are very much the same.

Now that we have a global drug-development effort, with pharmaceutical assets and networked suppliers stretching across the globe, harmonization of regulations has become more desirable, but not easier. Michael Zhou is a bioanalytical chemist who has had wide-ranging experience in pharmaceutical companies and CROs. He has worked across the globe in these roles and also as a consultant. His book covers the history and motivations behind regulated bioanalysis. He explains the alphabet soup of the jargon, the establishment of quality systems with recommendations on implementation, adhering to standards and being prepared for the inevitable regulatory inspections and audits (often from clients whose projects are at risk). He tells us how to evaluate instrumentation and select contract laboratories and to transfer methods and validate them.

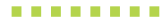 \\ "Michael Zhou is a bioanalytical chemist who has had wide-ranging experience in pharmaceutical companies and CROs."}

This volume also considers the technical aspects of well-performing SOPs and the many gotcha topics that can make a good method go bad. The focus is understandably on LC-MS and ligand-binding assays that dominate the field. Sample preparation strategies, automation/software, validation and cross-validation, improving sample throughput and LLOQs are covered and well referenced to the peerreviewed literature. Likewise, the applicable government regulatory guidances are also cited.

The last chapter includes an extensive glossary of terms that will be useful to both experts and beginners. There are five useful appendices: a check list for inspections/audits; a SOP template; a list of expected SOPs for regulated bioanalysis; a brief list of essential equipment;

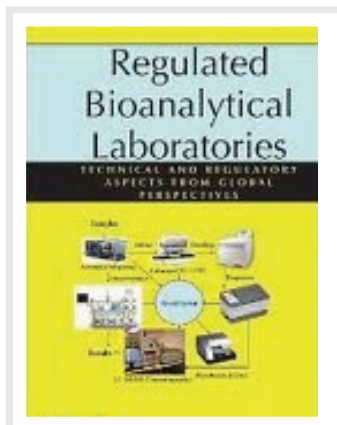

Regulated Bioanalytical Laboratories: technical and regulatory aspects from global perspectives

Author: Michael Zhou Publisher: Wiley

ISBN: 978-0-470-47659-8

\section{Peter T Kissinger}

Purdue University, 560 Oval Drive, West Lafayette, IN 47907, USA E-mail: kissingp@purdue.edu 


\section{News \& Analysis I Book Revew}

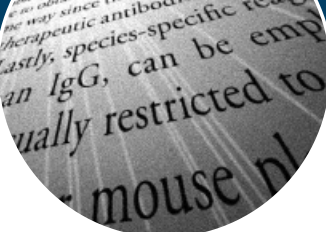

and a very extensive list of online resources from government and professional organizations. The index is satisfactory.

I have been using this volume for 3 months and have referred to it frequently. Zhou has done a good job that few others would attempt. I do not expect the movie rights to be sold soon. Regulatory science is still like watching paint dry, but we must get the best data that we can and, indeed, paint must dry.

\section{Financial \& competing interests disclosure}

The author has no relevant affliations or financial involvement with any organization or entity with a financial interest in or financial conflict with the subject matter or materials discussed in the manuscript. This includes employment, consultancies, honoraria, stock ownership or options, expert testimony, grants or patents received or pending, or royalties.

No writing assistance was utilized in the production of this manuscript. 